ARTICLE

\title{
Coherent multidimensional photoelectron spectroscopy of ultrafast quasiparticle dressing by light
}

\author{
Marcel Reutzel (10 1,2凶 , Andi Li ${ }^{1}$, Zehua Wang ${ }^{1} \&$ Hrvoje Petek (D) ${ }^{1 凶}$
}

Depending on the applied strength, electromagnetic fields in electronic materials can induce dipole transitions between eigenstates or distort the Coulomb potentials that define them. Between the two regimes, they can also modify the electronic properties in more subtle ways when electron motion becomes governed by time and space-periodic potentials. The optical field introduces new virtual bands through Floquet engineering that under resonant conditions interacts strongly with the preexisting bands. Under such conditions the virtual bands can become real, and real ones become virtual as the optical fields and electronic band dispersions entangle the electronic response. We reveal optical dressing of electronic bands in a metal by exciting four-photon photoemission from the $\mathrm{Cu}(111)$ surface involving a threephoton resonant transition from the Shockley surface band to the first image potential band. Attosecond resolved interferometric scanning between identical pump-probe pulses and its Fourier analysis reveal how the optical field modifies the electronic properties of a solid through combined action of dipole excitation and field dressing.

\footnotetext{
${ }^{1}$ Department of Physics and Astronomy and Pittsburgh Quantum Institute, University of Pittsburgh, Pittsburgh, Pennsylvania 15260, USA. ${ }^{2}$ Present address: I. Physikalisches Institut, Georg-August-Universität Göttingen, Göttingen, Germany. ${ }^{凶}$ email: marcel.reutzel@phys.uni-goettingen.de; petek@pitt.edu
} 
S pace-periodic arrangement of lattice ions in crystalline solids defines the $\boldsymbol{k}$-momentum dispersion of electronic quasiparticle bands. Light can interrogate such bands by stimulating electric dipole transitions. When a time-periodic optical field interaction exceeds all other perturbations, particularly at an optical resonance, however, it can also modify the system eigenstates. Then a quantum system is modified (dressed) by an optical field, it becomes a time crystal with novel, field-dependent electronic properties ${ }^{1}$. High-optical field control, which ultimately enables the rich physics of high-harmonic and attosecond pulse generation $^{2}$, then motivates the exploration of the light-wave electronics in solids ${ }^{3-9}$. Optical fields with designed electric field strength $\mathcal{E}$, and frequency $\omega_{l}$ can manipulate and control the quasiparticles in solids. Recent goals and achievements include light-induced superconductivity ${ }^{10}$, high-order nonlinearities ${ }^{3,6}$, the creation of Floquet topological bands in quantum materials ${ }^{9,11-19}$, and photoinduced phase transitions ${ }^{20,21}$.

Under perturbative conditions, light fields $\mathcal{E}(\tau)$ can excite multiquantum transitions from dispersive Bloch bands that are defined by the crystal structure, with their eigenstates specified by their momentum $\boldsymbol{k}$. An intense $\mathcal{E}(\tau)$ field, however, can perturb the system by causing Rabi flopping to occur between the optically coupled bands at frequencies $\tilde{\Omega}_{R}$ that can become comparable to that of the excitation field, $\omega_{l}$ (ref. ${ }^{13}$ ): the electronic bands are said to be dressed when the optical field amplitude and periodicity shifts their energies, and replicates them by integer photon quanta through the AC Stark and Floquet processes (Fig. 1e $)^{9,11}$. The optical dressing is thus marked by Autler-Townes (AT) splitting of solid-state bands into a new eigenstates $E_{ \pm}$(ref. ${ }^{22}$ ) separated by the generalized Rabi frequency

$$
\hbar \tilde{\Omega}_{R}(\tau, \boldsymbol{k})=\hbar \sqrt{\Omega_{R}^{2}(\tau)+\Delta^{2}(\boldsymbol{k})}
$$

where $\hbar \Omega_{R}(\tau)=\mu_{\text {eff }} \mathcal{E}^{n}(\tau)$ is the Rabi frequency of a $n$-photon transition, and $\hbar \Delta(\boldsymbol{k})$ is the $\boldsymbol{k}$-dependent detuning of Bloch bands ( $\hbar$ : the Planck constant). Moreover, we assume the dipole moment, $\mu_{\text {eff }}$, to be independent of $\boldsymbol{k}$ based on approximately symmetric photoemisson intensity distributions (with respect to + and - parallel momentum, $\left.k_{\|}\right)$, and $k_{\|}$-independent linear photoemission spectra of the initial band ${ }^{23}$.

Here, we apply intense ultrafast optical fields to a metal surface, a solid-state plasma, whose high electron density defines its quantum optical characteristics: attosecond screening and femtosecond electronic dephasing $24-26$. The ground and excited surface electronic bands of several metals like copper exist in projected band gaps that decouple them from stronger bulk interactions ${ }^{25}$ (Fig. 1d), making them inviting targets for probing their quantum optics by ultrafast multiphoton photoemission (mPP) spectroscopy and many-body theory ${ }^{25-30}$. We dress the surface electronic structure of $\mathrm{Cu}(111)$ with $\sim 20 \mathrm{fs}$ IR laser pulses by exciting the three-photon resonant transition from the occupied Shockley surface (SS) to the first image potential (IP1) band, and probe the coherent field interaction by further exciting photoelectrons into the photoemission continuum by interferometric-time-resolved multiphoton photoemission (ITR-mPP) spectroscopy (Fig. 1). The dressing and probing field $\mathcal{E}(\tau)$ combines identical, phase correlated pump-probe pulse pairs to excite four-photon photoemission (4PP). Advancing their delay (phase) in $\Delta \tau \sim 100$ as steps defines the optical fields with subfemtosecond precision, and simultaneously imaging photoelectron energy, $E_{f}$ and parallel momentum, $k_{\|}$, distributions records $E_{f}\left(k_{\|}, \tau\right)$-movies of coherent polarizations excited in the sample; the movies record the field-induced
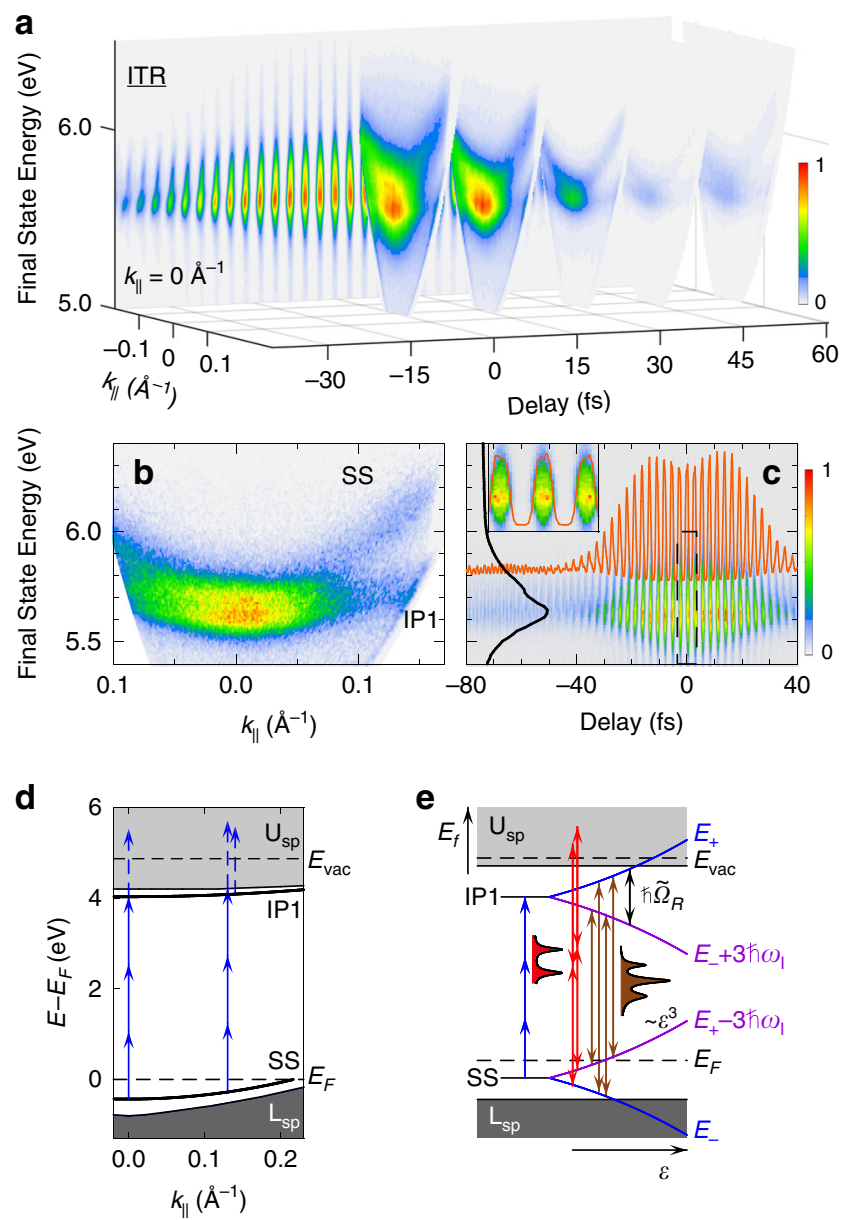

Fig. 1 Coherent photoelectron spectroscopy of optical dressing. a Threedimensional ITR-4PP movie of the three-photon resonant IP1 transition $\left(\hbar \omega_{l}=1.54-\mathrm{eV}\right)$. Each frame of Supplementary Movie 1, from which the data is taken, records the photoelectron signal counts vs. $E_{f}$ (relative to the Fermi level, $E_{F}$ ), and $k_{\|}$in $\sim 100$ as steps (sample movie frames are shown in $15 \mathrm{fs}$ intervals). The back panel shows a cross section through the movie for $k_{\|}=0 \AA^{-1}$. b Time-integrated 4PP spectrum showing the SS and IP1 bands for resonant excitation at $k_{\|}=$ $0 \AA^{-1}$. c Selection of data from a for $k_{\|}=0 \AA^{-1}$ showing an $E_{f}(\tau)$ interferogram; the profiles show $12 \mathrm{PC}$ trace for $E_{f}=5.65 \mathrm{eV}$ (orange) and a 4PP spectrum for $\tau=0$ fs (black); the inset expands the $E_{f}(\tau)$-data around $\tau=0$ fs (the dashed box). The dressing is evident in the dip in I2PC intensity at $\tau=0$ fs that is caused by the subfemtosecond splitting of the interference fringes, when the pump and probe fields are in-phase and $\mathcal{E}(\tau)$ is maximum, but not in the $\tau=0$ fs $E_{f}$-profile. $\mathbf{d}$ The unperturbed surface-projected band structure of $\mathrm{Cu}(111)$. The SS and IP1 bands (black lines) in the surface-projected band gap between the lower and upper spbands, $L_{s p}$ and $U_{s p}$ (gray shading), are coupled by the three-photon resonant laser field (blue); the fourth photon induces the photoemission (dashed blue). The excitation is resonant at $k_{\|}=0 \AA^{-1}$ producing one peak with enhanced 4PP yield in $\mathbf{b}$; the different $m_{\text {eff }}$ of the SS and IP1 bands, however, cause their detuning for increasing $k_{\|}$and consequent detection of two bands. e The optical dressing of the SS and IP1 bands: the increasing electric field $\mathcal{E}$ (right arrow) causes AC Stark effect shifts of the SS and IP1 bands to $E_{ \pm}$(blue), while the Floquet effect replicates $E_{ \pm}$ at integer multiples of $n \hbar \omega_{1}$ are (violet). The optical dressing is revealed by Mollow-Triplet structures in the resonant coherent polarizations at $3 \omega_{1}$ (brown arrows), and Autler-Townes doublets in the nonresonant coherent polarizations at $2 \omega_{l}$ (red arrows). 
quasiparticle dressing at the interface between the perturbative and non-perturbative light-matter interactions (Fig. 1a, Supplementary Movie 1). We explore how the optical field modifies the crystaldefined band structure on a sub-optical cycle timescale.

\section{Results}

Ultrafast quasiparticle dressing probed by coherent mPP. The $\mathcal{E}(\tau)$ field with $\hbar \omega_{l}=1.54-\mathrm{eV}$ excites the three-photon resonant transition from the SS to the IP1 band of $\mathrm{Cu}(111)$ at $k_{\|}=0 \AA^{-1}$. Different band dispersions of SS and IP1, which are defined by their band masses $\left(m_{S S}, m_{I P 1}\right)$, however, detune the resonant energy by $\hbar \Delta\left(k_{\|}\right)=\hbar^{2} / 2\left(m_{\mathrm{SS}}^{-1}-m_{\mathrm{IP} 1}^{-1}\right) k_{\|}^{2}$. The time-integrated $4 \mathrm{PP}$ spectrum in Fig. 1c confirms the SS and IP1 resonance ${ }^{29,31}$; such optical phase-integrated measurement, however, obscures their dressing, which, is encoded in the phase-resolved $E_{f}\left(k_{\|,} \tau\right)$ movies that reveal the electric field strength-dependent response (Fig. 1a, Supplementary Movie 1). To reveal the dressing, we first extract the $\mathcal{E}(\tau)$-dependent $4 \mathrm{PP}$ intensity for $k_{\|}=0 \AA^{-1}$ ( $\hbar \Delta=0 \mathrm{eV}$ ) by plotting the $2 \mathrm{D} E_{f}(\tau)$ data and, its time profile (1D interferometric two-pulse correlation (I2PC)) showing the photoelectron counts vs. $\tau$ at $E_{f}$ of the IP $1 \leftarrow$ SS resonance (Fig. 1c). If the optical field $\mathcal{E}(\tau)$ with approximately a Gaussian time profile generated the 4PP signal by only exciting dipole transitions, the I2PC trace in Fig. 1c would follow $\mathcal{E}^{8}(\tau)$ dependence, and therefore, sharply peak at $\tau=0$ fs (refs. ${ }^{30,32}$ ), but instead, it has a local minimum and retarded maxima at $\tau \approx \pm 15 \mathrm{fs}$, which portend the optical field dressing.

Fourier analysis of femtosecond field dressing of resonantly excited surface bands. The $E_{f}\left(k_{\|}, \tau\right)$-movie, therefore, incorporates both the $\mathcal{E}(\tau)$-dependent band dressing and quantum excitation pathways, which are revealed by its Fourier analysis. Fourier transformation (FT) of the 2D time-domain data in Fig. 1c resolves the induced polarization fields in the sample that oscillate at $1-4 \omega_{l}$ frequencies to produce the $4 \mathrm{PP}$ signal $^{30}$; in Fig. 2, we present the 2D-Fourier-filtered time-domain spectra of
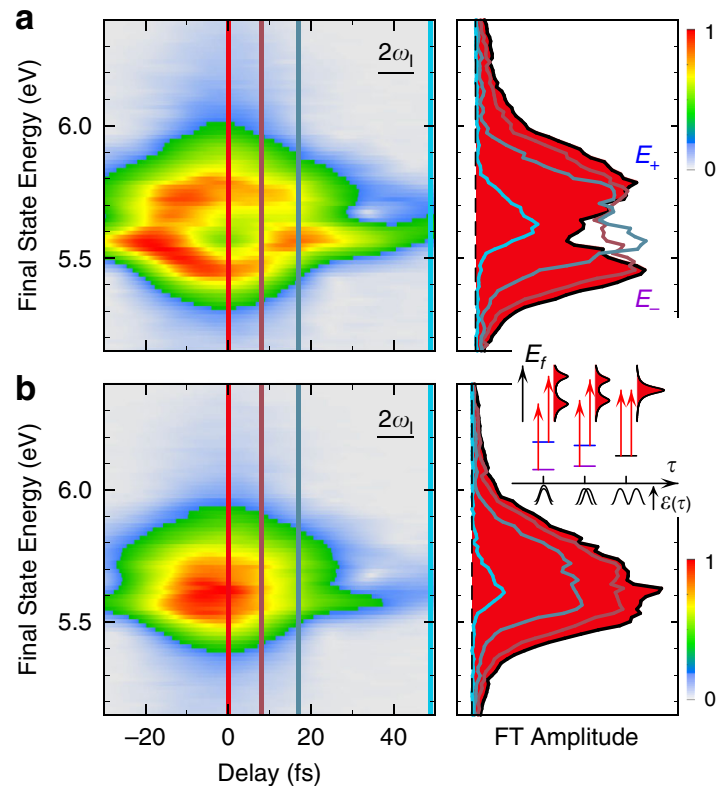

coherent polarizations oscillating at $2 \omega_{l^{-}}$and $3 \omega_{l^{-}}$-frequencies, which reveal the dominant pathways for how these coherences contribute to the 4PP signal, and thereby reveal the dressing. As detailed in Supplementary Note 3, the Fourier filtering involves the FT of the 3D ITR-4PP data to resolve the $1-4 \omega_{l}$ frequency components that contribute to the signal, followed by the selective inverse Fourier transformation (IFT) of each.

The $2 \omega_{l}$ and $3 \omega_{l}$ IFT spectra show peak doubling and tripling, respectively, near $\tau=0$ fs when the optical field amplitude is the largest (Fig. 2). The $3 \omega_{l}$-polarization represented by brown arrows in Fig. 1e reports how the optical field dresses the resonant bands causing components at $3 \omega_{l}$ and $3 \omega_{l} \pm \tilde{\Omega}_{R}$ frequencies to appear; such coherent polarizations produce Mollow-Triplet (MT) structures in gas-phase optical spectra ${ }^{22}$. By contrast, the nonresonant $2 \omega_{l}$-polarization, represented by red arrows in Fig. 1e, shows how the three-photon IP1 1 SS resonance dresses the interacting bands by revealing their AT doubling, when they are projected into the $E_{f^{-}}$continuum. The $2 \omega_{l^{-}}$and $3 \omega_{l^{-}}$spectra in Fig. 2, thus reveal the coherent subfemtosecond responses of the electronic bands to the dressing field. The $2 \omega_{l}$-spectrum shows that when $\tau=0 \mathrm{fs}$ and therefore, when $\mathcal{E}(\tau)=\mathcal{E}_{\text {pump }}(t)+$ $\mathcal{E}_{\text {probe }}(t+\tau)$ is maximum, at resonance the AT doublet splitting is $\hbar \tilde{\Omega}_{R}(\tau) \approx 0.3 \mathrm{eV}$, but as $|\tau|$ increases to $\sim 15 \mathrm{fs}$, the instantaneous $\mathcal{E}(\tau)$ field drops to recover the single undressed resonance. The dressing occurs within each optical cycle of $2.7 \mathrm{fs}$ as evidenced by the subfemtosecond splitting of the I2PC fringes near $\tau=0 \mathrm{fs}$ (inset of Fig. 1c), when constructive interference maximizes $\mathcal{E}(\tau)$. As expected for the AC Stark effect (Eq. (1)), the AT doublet structure disappears when $\mathcal{E}(\tau)$ amplitude is reduced by defocusing (Fig. 2b) or increasing $\tau$. Thus, the dressing and undressing follows the instantaneous $\mathcal{E}(\tau)$ strength, and is faster than the $\mathrm{Cu}(111)$ surface IP1 and SS band dephasing, which occurs by carrier scattering ${ }^{25}$ on $\sim 20 \mathrm{fs}$ timescale ${ }^{28,29}$.

Entanglement of in-plane momentum dispersion and photon dressing. A graphic feature that emerges from the ITR-4PP data
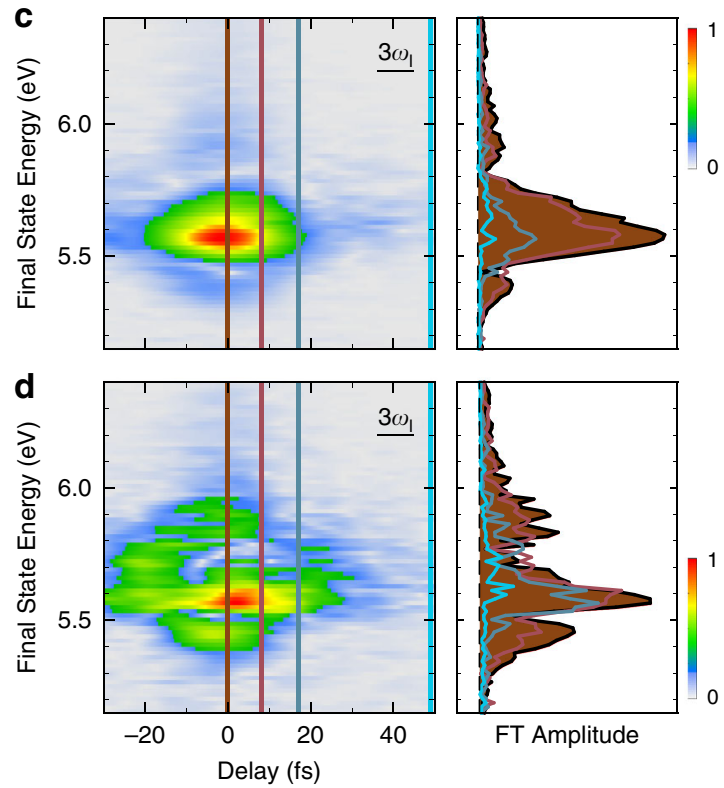

Fig. 2 Femtosecond $\mathcal{E}(\boldsymbol{\tau})$ field undressing of the surface bands. IFT spectra of the $2 \omega_{l}$ - $\mathbf{a}$, $\mathbf{b}$ and $3 \omega_{l}$-polarization $\mathbf{c}$, $\mathbf{d}$ responses at $k_{\|}=0 \AA^{-1}$ for excitation with high (top) and low (bottom) optical fluence. At high fluence and $\tau=0 \mathrm{fs}$, in $\mathbf{a}$ and $\mathbf{c}$, the AT doublet and the MT triplet structures are visible, respectively. As the instantaneous $\mathcal{E}(\tau)$ decreases with increasing $\tau$; however, the dressed bands converge to undressed ones within $\sim 15 \mathrm{fs}$ (cf. inset). At low fluence, $\mathcal{E}(\tau)$ is sufficient to drive the 4PP process, but its dressing is minimal. Energy line profiles taken for selected delay points (color coded) are shown for each measurement in the right. The inset shows how the dressing depends on the instantaneous excitation fields, as $\tau$ is increased. 

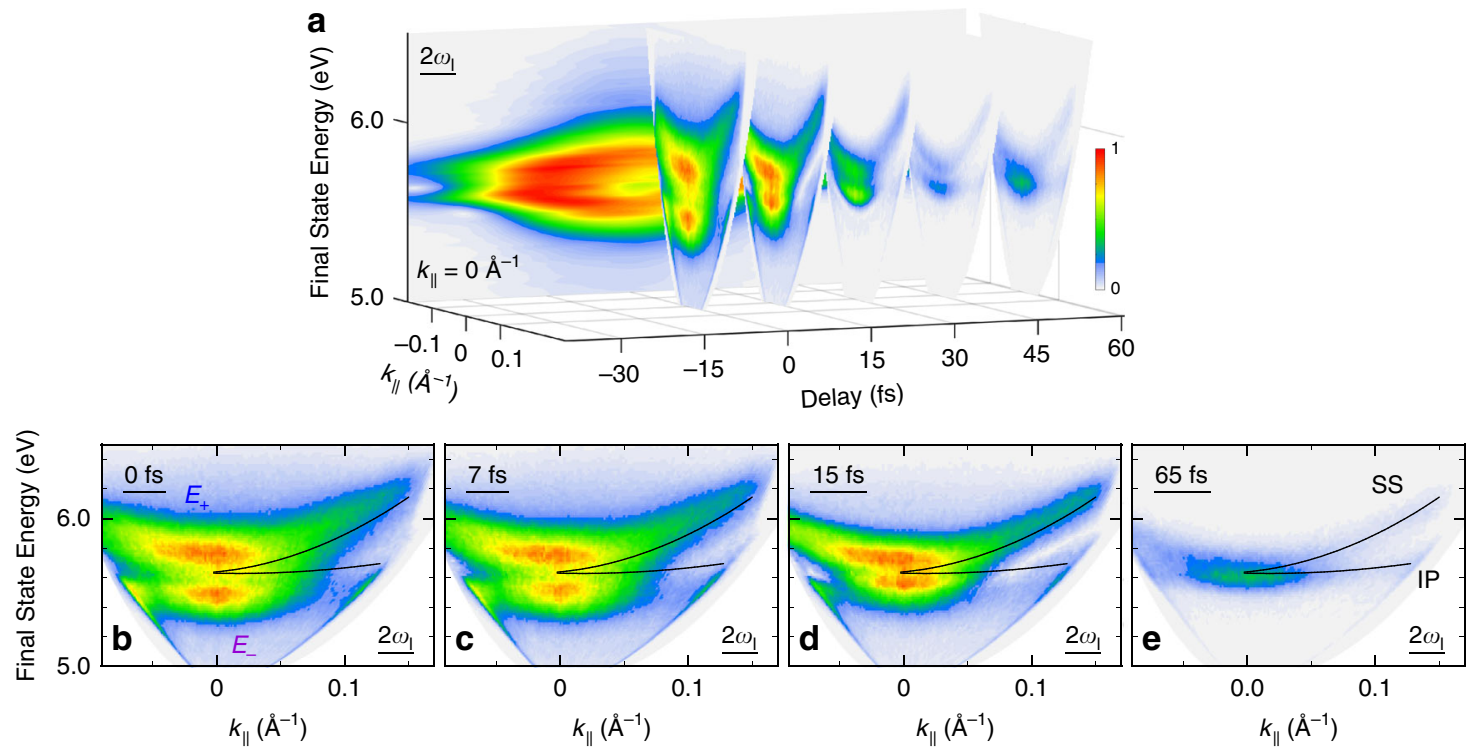

Fig. 3 Real-time movie of the $\boldsymbol{k}_{\| \mid}$-dispersion-optical field entanglement. a IFT of the $2 \omega_{l}$-polarization component in Supplementary Movie 1 (also, the Fig. 1a data) provides a 3D presentation of the $E_{f}\left(k_{\|}, \tau\right)$-resolved AT doublet (the individual movie frames show the $E_{f}\left(k_{\|}\right)$-spectra in $15 \mathrm{fs}$ intervals; the entire filtered data are presented in Supplementary Movie 2). The back panel is the $\tau$-dependence of the IFT $E_{f}\left(k_{\|}=0 \AA^{-1}\right)$ profile, such as in Fig. $2 a$. b-e $E_{f}\left(k_{\|}\right)$resolved snapshots for $\tau=0,7,15$, and 65 fs showing undressing as $\tau$ is increased. As a reference, the black lines indicate dispersions of the undressed SS and IP1 bands obtained at $\tau=100 \mathrm{fs}$ from the unfiltered ITR-4PP data, where the dressing is undetectable.

on $\mathrm{Cu}(111)$ is that $k_{\|}$-dispersion and optical dressing are entangled by the generalized Rabi frequency (Eq.(1)), which depends both on $\mathcal{E}(\tau)$ and $\hbar \Delta\left(k_{\|}\right)$. The entanglement is directly evident in the $2 \omega_{l}$-IFT-spectral component, which we extract from the data in Supplementary Movie 1, and examine as a function of $k_{\|}$and $\tau$ in Supplementary Movie 2. Supplementary Movie 2 conveys how the $E_{f}\left(k_{\|}\right)$-dependent polarization spectra, change as $\tau$ is advanced in $\Delta \tau \sim 100$ as steps to define the total $\mathcal{E}(\tau)$ field. The selected movie images in Fig. 3, highlight how changing $\mathcal{E}(\tau)$ and $k_{\|^{-}}$ dispersion affect the AT doublet dressing of the coupled bands. For $\tau=65 \mathrm{fs}$ (Fig. 3e), the pump and probe fields do not overlap, so the instantaneous $\mathcal{E}(\tau)$ is minimum, and the spectra reproduce the undressed $k_{\|}$-dispersions of the IP1 and SS bands (black lines). By contrast, for $15 \mathrm{fs} \lesssim|\tau|$ (Fig. $3 \mathrm{~b}-\mathrm{d}$ ), the pump and probe fields add coherently, sufficiently increasing $\mathcal{E}(\tau)$ to cause the $k_{\|^{-}}$ and $\tau$-dependent dressing, and thereby modify the band dispersions. As $\hbar \Delta\left(k_{\|}\right)$increases, however, the bands detune from resonance and therefore dressing diminishes causing them to converge to the undressed ones. This entanglement of band dispersion and dressing causes the quasiparticle band masses to become $\tau$-dependent. Thus, dressing can transform an electron into a hole band, potentially reversing the carrier transport in response to a femtosecond timescale optical field (cf. Supplementary Fig. 4), as could be evaluated by, for example, THzoptical photoemission spectroscopy ${ }^{8}$. Our coherent multidimensional movies show that light is a vector that can modify the electron mass, and physical properties that derive from it.

\section{Discussion}

Besides the dressing and $k_{\|}$-dispersion entanglement and dephasing, however, we must consider the dressing from a solidstate perspective. A quasiparticle in a solid is defined by its manybody interactions, specifically how a solid-state plasma responds to its Coulomb fields on a subfemtosecond screening timescale 24,31 . Specifically, the IP1 band forms through the manybody screening response of a metal ${ }^{26,32}$, and therefore, the screening and dressing temporal responses must be interdependent, as further theoretical and experimental scrutiny should elaborate.

Finally, we compare our results to the femtosecond two-color angle-resolved photoemission spectroscopy of Floquet bands in a $\mathrm{Bi}_{2} \mathrm{Se}_{3}$ topological insulator (see Supplementary Note 5 for details) and the transition metal dichalcogenide, $\mathrm{WS}_{2}$, in polarizationdependent optical spectroscopy. Gedik and coworkers ${ }^{16,33}$ demonstrated how time-coincident nonresonant mid-infrared excitation introduces new Floquet bands, and causes Bloch-Siegert band shifts. By contrast, here, we report the optical dressing under three-photon resonant conditions where the Stark shift dominates. We report the optical phase-resolved, subfemtosecond, $\mathcal{E}(\tau)$-dependent, entangled AT dressing through a nonlinear resonance between the equilibrium Bloch SS and IP1 bands. Such resonant dressing has only been reported for the fundamental semiconductor band gaps with alloptical $\boldsymbol{k}$-integrated techniques ${ }^{12-14}$.

Our 3D coherent photoelectron spectroscopy movies show that electronic bands are no longer defined just by the lattice potential, but applying a time-periodic external field introduces new Floquet bands, and causes shifts of nonlinearly excited resonant electronic bands through the AC Stark effect; specifically, breaking of the time-translation symmetry creates new nonequilibrium electronic structures within the solid state ${ }^{1}$, and opening of gaps between the time and space-derived bands entangles the space $\left(k_{\|}\right)$and time degrees of freedom, introducing frequency responses below that of the driving field. We stress that the interferometric-time- and angle-resolved $\mathrm{mPP}$ technique is particularly suited for recording of energy and momentum-resolved real-time movies of the fielddependent dressing of solids. Our multidimensional spectroscopy approach shows that optical dressing dynamics that may be obscured in time-integrated light intensity-dependent spectra, can become conspicuous by probing the induced coherences in electric field-dependent measurements. This experimental approach is applicable to all solid-state materials where momentum-dependent Floquet engineering might cause, for example, a time-dependent change of a material's electronic band topology 9,17 . Moreover, the entanglement between dressing and band dispersions provides the means to optically control the physical properties that depend on 
the quasiparticle band mass on a few femtosecond timescale. Our study demonstrates that quantum optics of the oldest optical material, a metallic surface, along with those of other high electron density solids, no longer reflect the passive coherent responses to optical fields, but can be actively controlled with subfemtosecond precision by nonlinear interactions with judiciously phase and amplitude tailored optical fields.

\section{Data availability}

The data that support the plots within this paper and other findings of this study are available from the corresponding author upon reasonable request

Received: 23 January 2020; Accepted: 14 April 2020;

Published online: 06 May 2020

\section{References}

1. Nayak, C. \& Yao, N. Y. Time crystals in periodically driven systems. Phys. Today 71, 40 (2018).

2. Corkum, P. B. \& Krausz, F. Attosecond science. Nat. Phys. 3, 381-387 (2007).

3. Ghimire, S. et al. Observation of high-order harmonic generation in a bulk crystal. Nat. Phys. 7, 138-141 (2011).

4. Schultze, M. et al. Controlling dielectrics with the electric field of light. Nature 493, 75 (2012).

5. Hohenleutner, M. et al. Real-time observation of interfering crystal electrons in high-harmonic generation. Nature 523, 572-575 (2015).

6. Langer, F. et al. Lightwave-driven quasiparticle collisions on a subcycle timescale. Nature 533, 225 (2016).

7. Higuchi, T., Heide, C., Ullmann, K., Weber, H. B. \& Hommelhoff, P. Lightfield-driven currents in graphene. Nature 550, 224 (2017).

8. Reimann, J. et al. Subcycle observation of lightwave-driven Dirac currents in a topological surface band. Nature 562, 396-400 (2018).

9. Oka, T. \& Kitamura, S. Floquet engineering of quantum materials. Annu. Rev. Condens. Matter Phys. 10, 387-408 (2019).

10. Mitrano, M. et al. Possible light-induced superconductivity in $\mathrm{K}_{3} \mathrm{C}_{60}$ at high temperature. Nature 530, 461-464 (2016).

11. Shirley, J. H. Solution of the Schrödinger equation with a Hamiltonian periodic in time. Phys. Rev. 138, B979-B987 (1965).

12. Fröhlich, D., Nöthe, A. \& Reimann, K. Observation of the resonant optical Stark effect in a semiconductor. Phys. Rev. Lett. 55, 1335-1337 (1985).

13. Mücke, O. D., Tritschler, T., Wegener, M., Morgner, U. \& Kärtner, F. X. Signatures of carrier-wave Rabi flopping in GaAs. Phys. Rev. Lett. 87, 057401 (2001).

14. Sie, E. J. et al. Valley-selective optical Stark effect in monolayer $\mathrm{WS}_{2}$. Nat. Mater. 14, 290 (2014).

15. De Giovannini, U., Hübener, H. \& Rubio, A. Monitoring electron-photon dressing in WSe 2 . Nano Lett. 16, 7993-7998 (2016).

16. Mahmood, F. et al. Selective scattering between Floquet-Bloch and Volkov states in a topological insulator. Nat. Phys. 12, 306-310 (2016).

17. Liu, H., Sun, J.-T., Cheng, C., Liu, F. \& Meng, S. Photoinduced nonequilibrium topological states in strained black phosphorus. Phys. Rev. Lett. 120, 237403 (2018).

18. Liu, H., Sun, J.-T. \& Meng, S. Engineering Dirac states in graphene: coexisting type-I and type-II Floquet-Dirac fermions. Phys. Rev. B 99, 075121 (2019).

19. Snoke, D. \& Littlewood, P. Polariton condensates. Phys. Today 63, 42-47 (2010).

20. Rohwer, T. et al. Collapse of long-range charge order tracked by time-resolved photoemission at high momenta. Nature 471, 490-493 (2011).

21. Frigge, T. et al. Optically excited structural transition in atomic wires on surfaces at the quantum limit. Nature 544, 207-211 (2017).

22. Fleischhauer, M., Imamoglu, A. \& Marangos, J. P. Electromagnetically induced transparency: optics in coherent media. Rev. Mod. Phys. 77, 633-673 (2005).

23. Dil, J. H., Meier, F. \& Osterwalder, J. Rashba-type spin splitting and spin interference of the $\mathrm{Cu}\left(\begin{array}{lll}1 & 1 & 1\end{array}\right)$ surface state at room temperature. J. Electron Spectrosc. Relat. Phenom. 201, 42-46 (2015).

24. Feibelman, P. J. Surface electromagnetic fields. Prog. Surf. Sci. 12, 287-407 (1982).
25. Echenique, P. M. et al. Decay of electronic excitations at metal surfaces. Surf. Sci. Rep. 52, 219-317 (2004).

26. Silkin, V. M., Lazić, P., Došlić, N., Petek, H. \& Gumhalter, B. Ultrafast electronic response of $\mathrm{Ag}(111)$ and $\mathrm{Cu}(111)$ surfaces: from early excitonic transients to saturated image potential. Phys. Rev. B 92, 155405 (2015).

27. Höfer, U. et al. Time-resolved coherent photoelectron spectroscopy of quantized electronic states on metal surfaces. Science 277, 1480 (1997).

28. Ogawa, S., Nagano, H., Petek, H. \& Heberle, A. P. Optical dephasing in $\mathrm{Cu}$ (111) measured by interferometric two-photon time-resolved photoemission. Phys. Rev. Lett. 78, 1339-1342 (1997).

29. Knoesel, E., Hotzel, A. \& Wolf, M. Temperature dependence of surface state lifetimes, dephasing rates and binding energies on $\mathrm{Cu}(111)$ studied with timeresolved photoemission. J. Electron Spectrosc. Relat. Phenom. 88-91, 577-584 (1998).

30. Reutzel, M., Li, A. \& Petek, H. Coherent Two-dimensional multiphoton photoelectron spectroscopy of metal surfaces. Phys. Rev. X 9, 011044 (2019).

31. Reutzel, M., Li, A., Gumhalter, B. \& Petek, H. Nonlinear plasmonic photoelectron response of $\mathrm{Ag}(111)$. Phys. Rev. Lett. 123, 017404 (2019).

32. Cui, X. et al. Transient excitons at metal surfaces. Nat. Phys. 10, 505-509 (2014).

33. Sie, E. J. et al. Large, valley-exclusive Bloch-Siegert shift in monolayer $\mathrm{WS}_{2}$. Science 355, 1066-1069 (2017).

\section{Acknowledgements}

The authors gratefully acknowledge financial support from DOE-BES Division of Chemical Sciences, Geosciences, and Biosciences grant no. DE-SC0002313. M.R. acknowledges support through the Feodor Lynen Fellowship Program from the Alexander von Humboldt Foundation.

\section{Author contributions}

M.R. and A.L. obtained the experimental data. M.R. analyzed the data. Z.W. contributed to data processing software development. M.R. and H.P. wrote the manuscript. H.P. conceived and directed the study. All authors discussed the results and their interpretation.

\section{Competing interests}

The authors declare no competing interests.

\section{Additional information}

Supplementary information is available for this paper at https://doi.org/10.1038/s41467020-16064-4.

Correspondence and requests for materials should be addressed to M.R. or H.P.

Peer review information Nature Communications thanks the anonymous reviewers for their contribution to the peer review of this work.

Reprints and permission information is available at http://www.nature.com/reprints

Publisher's note Springer Nature remains neutral with regard to jurisdictional claims in published maps and institutional affiliations.

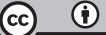

Open Access This article is licensed under a Creative Commons Attribution 4.0 International License, which permits use, sharing, adaptation, distribution and reproduction in any medium or format, as long as you give appropriate credit to the original author(s) and the source, provide a link to the Creative Commons license, and indicate if changes were made. The images or other third party material in this article are included in the article's Creative Commons license, unless indicated otherwise in a credit line to the material. If material is not included in the article's Creative Commons license and your intended use is not permitted by statutory regulation or exceeds the permitted use, you will need to obtain permission directly from the copyright holder. To view a copy of this license, visit http://creativecommons.org/ licenses/by/4.0/.

(C) The Author(s) 2020 